Meta

Journal des tradlucteurs

Translators' Journal

\title{
La traduction en Amérique latine
}

Compte rendu des SEDIFRALE 6, Buenos Aires (1987)

\section{Jean-Claude Gémar}

Volume 34, numéro 2, juin 1989

URI : https://id.erudit.org/iderudit/002838ar

DOI : https://doi.org/10.7202/002838ar

Aller au sommaire du numéro

Éditeur(s)

Les Presses de l'Université de Montréal

ISSN

0026-0452 (imprimé)

1492-1421 (numérique)

Découvrir la revue

Citer cette note

Gémar, J.-C. (1989). La traduction en Amérique latine : compte rendu des

SEDIFRALE 6, Buenos Aires (1987). Meta, 34(2), 322-324.

https://doi.org/10.7202/002838ar d'utilisation que vous pouvez consulter en ligne.

https://apropos.erudit.org/fr/usagers/politique-dutilisation/ 


\section{I - LA TRADUCTION EN AMÉRIQUE LATINE : SITUATION ET PERSEPCTIVES}

\section{A. PERSPECTIVES GÉNÉRALES}

En Amérique latine, les activités culturelles, scientifiques, industrielles et économiques sont aujourd'hui impossibles à appréhender et à gérer dans le seul cadre national. Comme dans le reste du monde, on assiste en effet dans tous les pays latinoaméricains à une accélération des flux de capitaux, de savoir-faire, d'idées et de produits, à une mobilité

\section{LA TRADUCTION EN AMÉRIQUE LATINE COMPTE RENDU DES SEDIFRALE 6 BUENOS AIRES (1987)}

Pour la sixième fois, les professeurs de français d'Amérique latine ont tenu, du 25 février au 6 mars 1987, leur rencontre traditionnelle organisée cette année par l'Association des professeurs universitaires de français d'Argentine (SAPFESU), à Buenos Aires.

Cette rencontre était scindée en deux grandes parties, dont la première était consacrée à la traduction, à son enseignement et à la formation de formateurs. Elle s'est déroulée sur trois jours, du 26 au 28 février, devant une assistance très nombreuse (plus de 850 personnes inscrites), compte tenu du sujet, la traduction, d'intérêt relativement secondaire par rapport à l'axe principal des SEDIFRALE, soit le français langue seconde - et son enseignement en Amérique latine.

Les activités organisées autour du thème de la traduction avaient attiré des participants de la plupart des pays d'Amérique latine et de la frạncophonie. Elles ont connu un succès considérable, alimenté par deux grandes tables rondes réunissant d'éminents spécialistes de la traduction - dont Marianne Lederer, Antoine Berman, Monique Legros - et par 13 ateliers qui se sont tenus pendant ces trois jours.

Ces rencontres ont drainé, des quatre coins du monde, francophone notamment, un nombre considérable de personnes et de spécialistes contribuant ainsi à élargir le débat et à l'élever. Elles constituent désormais un événement majeur dans la vie intellectuelle de la francophonie et des pays entièrement ou partiellement de langue française.

La meilleure façon de rendre justice aux rapporteurs des treize ateliers organisés dans le cadre des réflexions mences sur le thème de la traduction est de reproduire le document de synthèse générale. Chacun sera ainsi en mesure de juger par lui-même les constatations, commentaires et recommandations qui ont été faites, d'évaluer la situation de la traduction en Amérique latine et d'envisager ses perspectives d'évolution.

Jean-Claude Gémar Université de Montréal, Canada croissante des créateurs et à une internationalisation des productions culturelles.

Si les utilisateurs, ou les consommateurs finals, se trouvent être les citoyens d'un pays donné ils n'en sont plus les producteurs uniques ou privilégiés. Quelle que soit leur origine géographique, ces biens et ces services sont d'abord formulés dans la langue de leurs concepteurs ou de leurs producteurs. Le risque est grand que ces biens et services parviennent à leurs utilisateurs dans une langue étrangère ou, plus fréquemment, dans une version appauvrie de l'espagnol ou du portugais, ignorante des spécificités et des nuances nationales.

Il ne s'agit pas seulement là d'une grave atteinte à l'identité culturelle du citoyen latino-américain mais, à un niveau plus tangible, d'une déperdition d'information, génératrice de perte de temps, de qualité el de compétitivité. C'est le traducteur qualifíe qui se trouve être le jeteur de ponts entre producteurs et utilisateurs ou consommateurs de langues différentes.

C'est la qualité et la précision de son travail qui feront que les flux d'échanges soient réglés et leur contenu correctement assimilé par tous ceux auxquels ils sont destinés. Les mondes de l'administration, de la recherche et de la production sont particulièrement vulnérables à ce qu'on peut appeler la facilité de la non-traduction. L'information scientifique, technique ou commerciale, vćritable matière première de la recherche et de l'industrie, se présente souvent en langue étrangère. Pour cette raison, elle est parfois mal comprise ou ignorée, ce qui, face à la concurrence, se traduit par un handicap majeur. De la même manière, la promotion des produits ou services d'une même entreprise sur les marchés extérieurs est souvent faite dans une langue qui n'est pas celle de l'acheteur. Face aux entreprises qui ont compris qu'aujourd'hui, le client achète de préférence dans sa propre langue, celles d'entre elles qui pratiquent le monolinguisme ou le bilinguisme dans leurs catalogues, leur matériel promotionnel, leurs notices techniques, etc., se trouvent dès le départ en situation d'infériorité. L'investissement en traduction est aujourd'hui pour un organisme ou une entreprise un des éléments clés de ses gains de compétitivité. Il participe de la démarche plus générale de la recherche de la qualité : qualité des produits, de la communication et de l'image à l'externe. 
L'autarcie linguistique est donc en contradiction avec l'internationalisation des échanges. La traduction, c'est-à-dire la mise à la disposition de la totalité des produits scientifiques, culturels, administratifs ou industriels, dans autant de langues ou variantes de langues qu'il y a d'usagers ou de marchés, devient un impératif aussi bien économique que démocratique.

\section{B. SITUATION}

\section{LA FORMATION INITIALE DES TRADUCTEURS}

Il a été constaté qu'en Amérique latine, la formation initiale des traducteurs varie selon les pays :

dans certains pays, il n'existe pas de formation de base à la traduction;

dans d'autres, elle est dispensée immédiatement après l'obtention du diplôme d'études secondaires, sans concours d'admission préalable;

enfin, dans quelques pays, après l'obtention du diplôme d'études secondaires, on procède à une sélection par voie de concours.

D'autre part, le nombre des langues enseignées varie également. Il est apparu qu'il existe certaines carences au niveau des contenus des programmes de formation, en particulier dans les domaines de la méthodologie de la traduction, de la terminologie, de la documentation et de l'enseignement de la traduction spécialisée. Enfin, il a été noté que les enseignants des écoles de traduction ne sont pas souvent des traducteurs professionnels.

\section{LA FORMATION DES FORMATEURS}

Dans l'ensemble de l'Amérique latine, le besoin d'une formation des formateurs est impérieusement ressenti. En effet, au-delà de la formation initiale, aucune université ne propose de spécialisation ou de recyclage à l'intention des professeurs de traduction.

\section{LES OUTILS DU TRADUCTEUR}

Le traducteur latino-américain dispose à l'heure actuelle de certains outils comme les réseaux documentaires (centres, bibliothèques), les matériels lexicographiques (glossaires, dictionnaires spécialisés, etc.) et, au moins au Mexique et au Vénézuéla, dc banques de données terminologiques. Ces diverses ressources ne sont pas encore assez connues, ou restent difficiles d'accès. En ce qui concerne les moyens techniques de pointe mis de nos jours à la disposition des traducteurs (micro-ordinateurs, traitements de textes, systèmes de traduction assistee par ordinateur), leur coût paraît pour l'instant trop élevé en Amérique latine ; il en va de même pour l'accès aux grandes banques de données, techniquement possible, mais financièrement prohibitif. Néanmoins, les traducteurs latino-américains ne sauraient négliger l'importance de ces outils, et doivent envisager de les acquérir à moyen terme.

\section{LE MARCHÉ}

\section{a) Les marchés existants}

On a constaté que le marché de la traduction est en train de s'accroître en Amérique latine, ce qui explique en partie le besoin d'écoles de traduction. On a également noté que, souvent, les clients font appel à du personnel non qualifié, ce qui va au détriment de la qualité des traductions et, naturellement, de la situation des traducteurs professionnels. Enfin, le développement industriel en Amérique latine représente pour les traducteurs un vaste marché potentiel à exploiter.

\section{b) Stratégies pour l'élargissement du marché}

Il existe un projet d'enquête sur le marché de la traduction, émanant du S.I.I.T. (Servicio Iberoamericano de información sobre la traducción), dont le siège se trouve à Viia Ocampo en Argentine, et qui dépend de l'UNESCO. Cette enquête pourrait servir aux traducteurs en permettant:

de sensibiliser les entreprises à l'importance de la traduction en leur faisant voir les bénéfices qu'elles pourraient tirer d'informations qu'elles possèdent, mais n'exploitent pas entièrement;

de connaître le profil des entreprises, afin de savoir quel marché est visé :

de se renseigner sur l'utilisation que chaque entreprise fait des langues étrangères (langues employées, types d'emploi, documentation, etc.) ;

de connaitre les sources d'information en langues étrangères (catalogues, brevets, manuels, indices, etc.):

de s'adresser aux clients visés en tenant compte des variantes nationales de l'espagnol et du portugais :

d'adapter la traduction aux segmentations du marché ; et

de présenter les produits de l'entreprise dans la langue du client.

Dans le cas de l'Argentine, cet effort devrait être coordonné avec ceux du Secrétariat d'État à la science et à la technique, et, dans les autres pays, avec ceux des ministères chargés de la recherche, de la technologie, du commerce et de l'industrie.

\section{II - RECOMMANDATIONS}

Sur la base des conclusions des rapports des ateliers, le collectif «traduction» recommande

A. En ce qui concerne la formation des traducteurs, il convient : 
1) d'élargir l'accès à la profession de traducteur (avec contrôle au niveau de sortie);

2) de renforcer l'enseignement des langues préalable à la formation des traducteurs en créant des cours gratuits pris en charge par les universités et les instituts supérieurs;

3) d'introduire dans les cursus universitaires une troisième langue, voire une quatrième ;

4) d'introduire dans les cursus l'enseignement de la terminologie, de la documentation, de la rédaction spécialisée, de la révision et des techniques informatiques;

5) de créer des centres de formation à la traduction dans les pays ou régions où il n'en existe pas, si le besoin s'en fait sentir ;

6) de constituer des fonds documentaires relatifs à la traduction (manuels, etc.) et de traduire en espagnol et en protugais des ouvrages sur la traduction et son enseignement ;

7) d'organiser des stages en entreprise, etc. ;

8) de renforcer les cours de traduction spécialisée technique et scientifique (niveau maitrise) sur la base d'une formation de traducteur généraliste ;

9) de combattre la rigidité des écoles de traduction et de stimuler l'esprit d'initiative des étudiants;

10) de confier l'enseignement de la traduction de préférence à des personnes ayant une expérience professionnelle de la traduction;

11) d'encourager la recherche en traductologie (travaux interdisciplinaires);

12) de créer des bourses destinées aux traducteurs, formateurs et étudiants ;

13) de mettre à l'étude la création d'une école latino-américaine de traduction (projet de l'Université de la Plata, Argentine);

14) de promouvoir une collaboration organique entre les écoles de traduction latino-américaines ;

15) de promouvoir des échanges au niveau international, en particulier dans.le monde francophone:

a) stages, séminaires, cours de spécialistes étrangers en Amérique latine (traduction, terminologie, etc.) ;

b) stages, séminaires, etc. de traducteurs, formateurs, latino-américains à l'étranger.

\section{B. Autres recommandations :}

16) créer un Bulletin d'information latino-américain sur la traduction et les disciplines connexes ;

17) intensifier et coordonner les activités des associations de traducteurs en Amérique latine, et en créer dans les pays qui n'en possèdent pas;

18) développer des campagnes de promotion de la traduction destinées

a) au grand public

b) aux commanditaires (éditeurs, entreprises, organismes d’État, etc.);
19) créer des prix nationaux et internationaux de traduction;

20) éditer un dépliant sur le traducteur et les enjeux de la traduction;

21) lutter pour la reconnaissance du droit d'auteur de traducteurs ;

22) mettre à l'étude la création de réseaux de consultation terminologique et documentaire ;

23) promouvoir des actions de concertation avec le S.I.I.T.;

24) lancer des études de marché sur la traduction et définir des stratégies de développement des activités de traduction en Amérique latine. 